\title{
Management of infertile women with mosaic Turner syndrome by using homologous oocyte
}

\author{
Ramazan Ozyurt' ${ }^{1}$, Nurettin Turktekin², Ozan Ozolcay' ${ }^{1}$, Cemil Karakus ${ }^{3}$ \\ IIstanbul IVF-Centre, Istanbul, Turkey \\ ${ }^{2}$ Vocational School of Health Services, Nişantaşı University, Istanbul, Turkey \\ ${ }^{3}$ Vocational School of Health Services, Beykent University, Istanbul, Turkey
}

\begin{abstract}
Introduction: The aim of the study was to investigate the effect of the use of homologous intracytoplasmic sperm injection on clinical pregnancy and live birth rates in infertile patients with mosaic Turner syndrome (TS).

Material and methods:

Twelve infertile cases who had unsuccessful in vitro fertilization (IVF)/intracytoplasmic sperm injection (ICSI) trials and were diagnosed with mosaic TS were included in the study. Twelve cases with unexplained infertility were selected as the control group. The diagnosis of TS was made according to the TS guideline. Karyotype identification was made by fluorescence in situ hybridization method (FISH) analysis of peripheral blood samples. The patients whose first cell line consisted of 45,XO and second cell line consisted of any karyotype were accepted as mosaic TS. TS cases with gonadal dysgenesis or classical TS were not included in the study. Homologous intracytoplasmic sperm injection was performed in both groups of patients. While embryo transfer was performed after pre-implantation genetic diagnosis (PGD) in 5 patients, embryos were transferred without PGD in 7 patients. Clinical pregnancy and live birth rates of the patients in both groups were compared.

Results: Age and body mass index values of both groups were found to be similar. The number of unsuccessful IVF attempts and duration of infertility were similar in both groups. However, the total dose of recombinant follicle stimulating hormone ( $\mathrm{rFSH}$ ) was higher and the duration of $\mathrm{rFSH}$ use was longer in TS patients. The total number of oocytes collected and metaphase II stage (MII) oocytes were significantly lower in the TS group. The 45,X/46,XX karyotypes detected in 6 of 12 cases were recorded as the most frequent mosaic TS pattern. The mosaic form we detected as the second most frequent was 45,X/46,XX/47,XXX karyotype seen in 3 cases. Approximately half of the cases had 45,X/46,XX karyotype, while one fourth had 45,X/46,XX/47,XXX. The remaining 3 cases were the rarer mosaic TS forms. There was no significant difference between the mosaic TS group and the control group in terms of clinical pregnancy rates $(58.3 \%$ vs. $50 \%, p<0.55)$. Live birth rates were found to be significantly higher in the control group compared to the TS group $(57.1 \%$ vs. $83.3 \%, p<$ 0.001 ). Abortion rates were $42.8 \%$ in the TS group and $16.6 \%$ in the control group. The abortion rate in the TS group was approximately 2.6 times higher than in the control group $(p<0.02)$. One of the three miscarriages was from the PGD group (33.3\%), and the remaining two were from the non-PGD group (66.6\%).

Conclusions: Before an infertile patient with mosaic TS is referred for donation, IVF/embryo transfer (ET) should be planned with her own eggs and by applying PGD.

Key words: mosaic Turner syndrome, homologous oocyte, clinical pregnancy, live birth, miscarriage.
\end{abstract}




\section{Introduction}

Turner syndrome (TS) is the most common chromosomal anomaly in women, characterized by the complete or partial absence of one of the X chromosomes [1]. In most of the cases, the missing $\mathrm{X}$ chromosome is of paternal origin and occurs once in 2500 live births as a result of the defect in meiotic division $[2,3]$. Haploinsufficiency of X chromosomes causes the developmental defect of the gonads, which is the main finding of the syndrome [4]. Dysgenetic gonads are more common in classical TS $(45, \mathrm{X})$ cases where the $\mathrm{X}$ chromosome is completely absent. In mosaic cases, the gonads are more prominent thanks to the $\mathrm{X}$ chromosomes found in other karyotypes $(45, \mathrm{X} / 46, \mathrm{XX})$. Thanks to the second $\mathrm{X}$ chromosome in mosaic cases, pubertal development and follicle development can be completed and spontaneous pregnancies can occur [5]. Although pregnancy is reported in classical cases, it is significantly low compared to mosaic cases $[5,6]$. Due to increased follicular atresia in TS cases, the patient may lose her ovaries before pubertal development is completed [3]. Premature ovarian failure is a common problem in TS patients whose pubertal development is completed [5] Therefore, these patients have very little time to get married and have children. Rapid ovarian tissue cryopreservation is of critical importance in the group of patients who are not currently considering a baby [7]. The development of oocyte and ovarian tissue vitrification has allowed many women with TS to achieve pregnancy at a young age. Unfortunately, follicles were found in the cortex only in one-fourth of the patients who underwent ovarian cortical biopsy [7].

In classical TS cases, fertility treatment is usually performed with oocyte donation, while in mosaic cases with gonads, in vitro fertilization (IVF)/intracytoplasmic sperm injection (ICSI) can be performed using homologous oocytes. There are also mosaic TS forms with a high spontaneous pregnancy rate [6]. When the literature was reviewed, the rate of patients with TS who used donation and/or homologous oocyte and delivered term was reported as $38 \%$ [6]. Unfortunately in mosaic TS cases, the chances of spontaneous pregnancy and giving live birth to a baby are very low and are estimated as $2 \%$ [8]. However, the number of studies in which IVF/ICSI was performed using homologous oocytes and pregnancy was reported is very few and consists of case reports. Our study was planned to compare the clinical, laboratory and demographic data of 12 patients who were diagnosed with mosaic TS and underwent IVF/ICSI with homologous oocytes with patients with normal karyotypes $(46, \mathrm{XX})$ and unexplained infertility.

Our study is clinically important in terms of both the number of women with TS undergoing IVF/ICSI with homologous oocytes and the comparison of the results with the group with a normal karyotype.

\section{Material and methods}

Twelve female patients who had infertility complaints and were diagnosed with mosaic TS were included in the study. Participants diagnosed with mosaic TS were selected from among 23 patients who were admitted with infertility complaints and for whom karyotype analysis was performed. Mosaic TS diagnosis was made according to TS guidelines [9]. Presence of the abnormal karyotype was accepted as the main criterion in the diagnosis of mosaic TS. In addition, the patient's phenotypic appearance, pubertal history, primary and/or secondary amenorrhea were recorded. Twelve patients with unexplained infertility diagnosed as age and body mass index (BMI) matched with the TS group were selected as the control group. The women in the control group were selected from among patients whose karyotypes were 46,XX. The subjects who participated in the study were selected among the patients who applied to Istanbul IVF-Centre with the complaints of infertility between 2016 and 2020. Patients with classical TS were not included in the study. Some of the patients were diagnosed with TS when they applied to us/came to our clinic (Istanbul IVF-Center). Some patients were diagnosed with TS during further examinations performed in our clinic. TS was diagnosed as a result of the genetic tests we performed in patients with a phenotypically different appearance, decreased ovarian reserve or recurrent implantation failure. No remarkable finding was found in the physical examination of the patients with mosaic TS. In cases with classical TS, there was a characteristic phenotype image including short stature and gonadal dysgenesis, but these cases were excluded from the study.

Cytogenetic examination for the diagnosis of TS was made by using peripheral blood samples which shows the numerical or structural abnormalities of the sex chromosomes. Fluorescence in situ hybridization method (FISH) was used for diagnosis of mosaic forms. For FISH analysis, 100 cells in metaphase were evaluated and the karyotype of the patients was determined. The fraction of normal and abnormal karyotype cell rates was determined for each patient. Patients with the fraction of abnormal karyotype cells above $5 \%$ were diagnosed with TS if they simultaneously had other normal or abnormal karyotypes or any of the isochromosome Xq karyotypes. Mosaic TS was detected in 12 of 23 cases, who had karyotype analysis with suspicion of chromosomal anomaly, and classical TS was detected in 2 cases. In the remaining 9 cases, karyotypes were reported as normal. None of the mosaic cases had a history of spontaneous pregnancy or IVF pregnancy. Because of the increased risk of cardiovascular disease in TS cases, all of the cases were evaluated by cardiological examination. Patients with aortic coarctation or bicuspid aortic valve were excluded from the study. Patients who did not allow examination by cardiologists were also excluded from the study.

In addition to basal hormonal levels, pelvic examination and transvaginal ultrasound findings were assessed and the antral follicle count was recorded. Oestrogen-based hormone therapy was used for several months before IVF/ICSI in patients with low ovarian reserve and/or insufficient uterine and endometrial development. Women with mosaic TS were treated according to a standard antagonist protocol with individually dosed recombinant follicle-stimulating hormone (FSH) starting on day 2-3 of the menstrual cycle. Gonadotrophin-re- 
leasing hormone antagonist was started on the $5^{\text {th }}$ or $6^{\text {th }}$ day of stimulation. When at least three follicles reached 16-17 $\mathrm{mm}$ in diameter, maturation of follicles was induced with recombinant human chorionic gonadotropin (hCG) (Ovitrelle, Merck-Serono, $250 \mathrm{mg}$, Modugno, BA, Italy). Oocyte collection was performed $36 \mathrm{~h}$ after hCG application. Ovarian follicles were aspirated using a single-lumen, 17-gauge needle guided by trans-vaginal ultrasonography. Oocyte pooling was performed in cases where insufficient oocyte was collected on the day of oocyte pick-up (OPU). While embryo transfer was performed after pre-implantation genetic diagnosis (PGD) in 5 patients, embryos were transferred without PGD in 7 patients. Normal karyotype was found in all 5 patients with TS who underwent PGD. One or two good quality or grade 1-2 embryos were transferred on the $3^{\text {rd }}$ or $5^{\text {th }}$ day of ICSI. While frozen embryo transfer (ET) was applied to 5 cases in the PGD group, fresh ET was applied to 7 women in the non-PGD group. Four of seven women in the non-PGD group were applied fresh ET on the $3^{\text {rd }}$ day, the remaining 3 were applied on the $5^{\text {th }}$ day. Embryo transfer was performed on the fifth day in women undergoing PGD.

Micronized progesterone was initiated vaginally for luteal support. Estradiol valerate treatment was continued 3 times a day. Serum $\beta$-hCG levels were measured in all patients on the $12^{\text {th }}$ day of embryo transfer. In the presence of a positive pregnancy test, luteal support was continued and USG was performed in the $4^{\text {th }}$ week of the transfer and the presence of gestational sac and thus clinical pregnancy was confirmed. Clinical pregnancy rate was defined as evidence of a gestational sac, confirmed by ultrasound examination. Live birth rate was defined as delivery of a live foetus after 24 completed weeks of gestational age. All subjects gave their informed consent to inclusion before they participated in the study.
The study was performed according to the guidelines of the Declaration of Helsinki on human experimentation and was approved by the Local Ethics Committee.

\section{Statistical analysis}

The Statistical Package for Social Sciences, version 23.0 (SPSS Inc., Chicago, IL, USA) was used for statistical analysis. Demographic and other individual group parameters were assessed with one-sample Kolmogorov-Smirnov $Z$ test and were found abnormally distributed. Statistical comparisons between mosaic TS and control groups were performed by non-parametric Mann-Whitney $U$ test. Data are presented as mean \pm SD. For all comparisons, statistical significance was defined by $p<0.05$.

\section{Results}

The mean age of the patients in the mosaic TS and control group was $28.4 \pm 1.3$ and $27.5 \pm 0.4$, respectively. Age and BMI values of both groups were found to be similar. The number of unsuccessful IVF attempts and duration of infertility were similar in both groups. However, the total dose of recombinant follicle stimulating hormone ( $\mathrm{rFSH}$ ) was higher and the duration of rFSH use was longer in TS patients. The total number of oocytes collected during OPU and MII oocytes were significantly lower in the TS group. Other laboratory and demographic parameters of the patients in both groups are shown in Table I. Endometrial thickness and E2 levels on the day of hCG were found similar in both groups. Basal FSH, LH and E2 levels measured on day 3 of the cycle of all participants were in the normal ranges.

During the karyotype analysis of participants we detected different mosaic TS patterns with second cell lines (Table II). The 45,X/46,XX karyotype detected in 6 of 12 cases were recorded as the most frequent mosaic TS pattern. The mo-

Table I. Comparison of demographic and reproductive parameters of women with TS and the control group

\begin{tabular}{|l|c|c|c|}
\hline Parameter & Mosaic TS $(n=12)$ & Unexplained infertility $(n=12)$ & $P$-value \\
\hline Age [years] & $34.3 \pm 1.3$ & $33.5 \pm 0.4$ & 0.07 \\
\hline BMI $\left[\mathrm{kg} / \mathrm{m}^{2}\right]$ & $28.2 \pm 1.9$ & $27.7 \pm 0.3$ & 0.40 \\
\hline Day 3 FSH [IU/l] & $6.81 \pm 2.03$ & $6.67 \pm 3.02$ & 0.08 \\
\hline Day 3 LH [IU/l] & $7.10 \pm 1.10$ & $6.60 \pm 0.12$ & 0.33 \\
\hline Day 3 E2 [pg/ml] & $55.1 \pm 5.11$ & $53.2 \pm 6.01$ & 0.06 \\
\hline Failed IVF attempts & $1.83 \pm 0.4$ & $1.86 \pm 2.0$ & 0.20 \\
\hline Infertility duration & $5.66 \pm 1.8$ & $4.97 \pm 0.8$ & 0.08 \\
\hline Total oocyte & $6.02 \pm 2.3$ & $11.03 \pm 4.0$ & $0.002^{*}$ \\
\hline MII oocyte & $5.12 \pm 3.8$ & $8.03 \pm 3.2$ & $0.001^{*}$ \\
\hline No. of embryos formed & $3.12 \pm 0.2$ & $5.32 \pm 3.9$ & $0.02^{*}$ \\
\hline No. of embryos transferred & 1 & 1 & NA \\
\hline PGD, $n$ (\%) & $5(41.6)$ & 0 & NA \\
\hline The number of clinical pregnancy, $n$ (\%) & $7(58.3)$ & $6(50)$ & 0.55 \\
\hline The number of live birth, $n$ (\%) & $4(57.1)$ & $5(83.3)$ & $0.01^{*}$ \\
\hline Miscarriage rate, $n$ (\%) & $3(42.8)$ & $1(16.6)$ & $0.02^{*}$ \\
\hline
\end{tabular}

${ }^{*} p<0.05$, PGD - pre-implantation genetic diagnosis, NA - not applicable. Data are presented as mean \pm SD. 
Table II. Homologous IVF/ICSI outcome of 12 women with mosaic TS according to their karyotypes

\begin{tabular}{|l|c|c|c|c|}
\hline Patients & Mosaic TS karyotype $(n=12)$ & Clinical pregnancy & Live birth & Miscarriage \\
\hline 1 & $45, X / 46, X X$ & 1 & 1 & 0 \\
\hline 2 & $45, X / 46, X X$ & 0 & 0 & 0 \\
\hline 3 & $45, X / 46, X X$ & 0 & 0 & 0 \\
\hline 4 & $45, X / 46, X X$ & 1 & 1 & 1 \\
\hline 5 & $45, X / 46, X X$ & 1 & 0 & 0 \\
\hline 6 & $45, X / 46, X X$ & 1 & 0 & 0 \\
\hline 8 & $45, X / 47, X X X$ & 1 & 0 \\
\hline 10 & $45, X / 47, X X X$ & 1 & 1 \\
\hline 11 & $45, X / 46, X X / 47, X X X$ & 1 & 0 \\
\hline 12 & $45, X / 46, X X / 47, X X X$ & 0 & 0 \\
\hline Total & $45, X / 46, X X / 47, X X X$ & 1 & 0 & $3(42.8 \%)$ \\
\hline
\end{tabular}

saic form we detected as the second most frequent was 45,X/46,XX/47,XXX karyotype seen in 3 cases. Approximately half of the cases had $45, \mathrm{X} / 46, \mathrm{XX}$ karyotype, while one fourth had 45,X/46,XX/47,XXX. The remaining 3 cases were the rarer mosaic TS forms. Clinical pregnancy was detected in 7 (58.3\%) cases after IVF/ICSI was performed in 12 mosaic TS patients, while pregnancy could not be achieved in the remaining 5 cases. Of the 7 patients who conceived, $4(57.1 \%)$ had a live birth and $3(42.8 \%)$ patients had abortion. One of the three miscarriages was from the PGD group (33.3\%), and the remaining two were from the non-PGD group (66.6\%). When the cases were analysed according to the mosaic karyotypes, 4 of 6 cases with 45,X/46,XX karyotypes conceived after IVF/ICSI, but two of them were able to deliver live birth. Only one of the two cases with 45,X/47,XXX karyotype gave a live birth and one case did not conceive. Two of the 3 cases with $45, \mathrm{X} / 46, \mathrm{XX}$ /47,XXX karyotype conceived, but only one case was able to give a live birth, and one of them had an abortion. On the other hand, pregnancy could not be achieved in woman with 45,X/46,XX/47, XXX/48,XXXX karyotype.

While clinical pregnancy $(50.0 \%)$ was found in 6 patients in the control group, pregnancy could not be achieved in 6 patients. Five $(83.3 \%)$ of 6 pregnant women gave live births and $1(16.6 \%)$ case resulted in abortion. In terms of clinical pregnancy rates, there was no difference between the mosaic TS group and the control group (58.3\% vs. $50 \%, p<$ $0.55)$. Live birth rates were found to be significantly higher in the control group compared to the TS group (57.1\% vs. $83.3 \%, p<0.001)$. Abortion rates were $42.8 \%$ in the TS group and $16.6 \%$ in the control group. The abortion rate in the TS group was approximately 2.6 times higher than in the control group $(p<0.02)$. Since abnormal phenotypic appearance was found in a newborn, karyotype analysis was performed and found normal. Cardiology consultation was performed in all newborn babies and normal echocardiographic findings were found.

\section{Discussion}

Even though patients with mosaic TS are in a better condition in terms of pubertal development, ovarian reserve and spontaneous pregnancy compared to classical TS, they do not have much time to have a baby. Even if approximately half of mosaic TS patients complete their pubertal development and enter the reproductive period, they are at risk of early menopause [10]. Although there is an approximately $8 \%$ chance of spontaneous pregnancy, this rate is not sufficient for many patients to become pregnant [11]. Although the cases who have ovarian tissue cryopreservation in the prepubertal period seem more lucky in terms of pregnancy, not every cortex tissue may contain enough follicles. The number of follicles to be obtained varies according to the patient's karyotype and ovarian reserve [7]. Most of the mosaic TS cases who reach adulthood can conceive using one of the assisted reproductive techniques despite their decreased ovarian reserves [12]. In most cases with early menopause, oocyte donation is required. However, many patients do not like oocyte donation for different reasons.

When the literature was reviewed, there were reports of TS cases who became pregnant spontaneously as isolated case reports, as well as mosaic TS cases who underwent IVF/ICSI using homologous oocytes and became pregnant [4]. Our study is important in that it is a large series that compares IVF/ICSI results using homologous oocytes in 12 mosaic TS patients and IVF/ICSI results of cases with normal karyotype and unexplained infertility. While clinical pregnancy was detected after IVF/ICSI was performed with homologous oocyte in 7 (58\%) of 12 TS cases, pregnancy was detected in 6 (50\%) cases in the control group. Despite the lower total oocyte counts in the TS group, we could not detect any difference in clinical pregnancy rate between the two groups. However, live birth rates were significantly lower in the TS group. While 4 (57\%) out of 7 patients with clinical pregnancy in the TS group had a live birth, 5 (83\%) 
patients in the control group had a live birth. Live birth rates were significantly higher in the control group than in the TS group. Despite the low total oocytes and metaphase II stage (MII) oocytes in the TS group, clinical pregnancy rates were similar to the control group, suggesting that the oocytes in TS patients are genomically healthy. However, the low rate of live births in patients with TS weakens our idea of the presence of healthy embryos to some extent. As the pregnancy progresses, the genetic defect in the embryo may become more obvious. In addition, there may be a problem with the implantation. Since the endometrial thickness was morphologically similar between the two groups, the decreased live birth rates in the TS group may possibly be related to embryo quality. Since the number and quality of embryos transferred was similar in the groups, the main problem is more likely to be related to the genomic content of the embryo. Since the semen analysis of the male partners of the patients in both groups was normal, the possible defect in embryo quality is more likely to be related to the oocytes. When the literature is reviewed, live birth rates of patients with TS were reported as $38 \%$. In our study, live birth rates were found to be $57 \%$ [6]. The reason for the higher live birth rates in our study compared to the literature may be due to the low number of cases participating in the study.

The fact that the miscarriage rates in our patient group with TS were approximately 2.6 times higher than in the control group, strengthens the possibility of genetic problems related to oocytes in embryos. When the literature was reviewed, it was reported that the abortion rate in TS patients was $29 \%$ [3]. In our patients, the abortion rate was found to be approximately $42 \%$. Three of the 12 patients with TS had abortion. One (33.3\%) of the three abortion cases was from the PGD group, and the remaining 2 (66.6\%) were from the non-PGD group. The abortion rate in patients who did not undergo PGD was two times higher than in those who underwent PGD. However, it was an obvious finding that abortion rates increased in patients with TS, independent of PGD. When compared with the control group, the rate of abortion was 2 times higher in those who had PGD, while this rate was up to 3 times higher in those who did not have PGD. The significant increase in the incidence of $X$ chromosome anomalies such as X monosomy and structural defects after IVF/ICSI using homologous oocyte also explains the miscarriage rates in our series $[3,13]$. Concordantly, it has been reported that the rates of miscarriage, stillbirths, or congenital defects are significantly higher in spontaneous or IVF/ICSI pregnancies in mosaic TS patients [5]. There are two possible reasons why our abortion rates (42\%) are higher than rates reported in the literature (29\%). First, since PGD was not performed in all cases, genetically problematic embryo transfer may have been performed. The second possibility is that donation cycles are among the abortion rates in the literature. Therefore, since the abortion rate will be low in young and healthy eggs used for donation, we can see relatively increased abortion rates in our series.

Karyotype analysis was performed due to suspicious phenotypic appearance in one of the four women who gave live birth and it was found to be normal. Karyotype analysis was not performed in the other three newborns. Consistent with our results, the study group detected different types of chromosomal anomalies in 6 out of 26 cases who gave birth with the diagnosis of mosaic TS [14]. However, since most of the mosaic TS cases have a phenotypically normal appearance, it is not very accurate to look only at the phenotype when making a karyotype decision [4].

If a patient with mosaic TS applied for treatment before puberty, ovarian tissue cryopreservation should be recommended. If the patient applied in adulthood, the karyotype should be confirmed by different analyses from regions such as blood, buccal mucosa and urine. In patients with confirmed TS, IVF/ICSI with the patient's own oocytes should be recommended before recommending donation based on ovarian reserve. The clinical pregnancy rates in IVF/ICSI performed with the patient's own egg similar to healthy controls, should encourage the clinician to use homologous oocytes. Although live birth rates are lower than in the control group, IVF/ICSI should be performed with their own eggs before donation in patients with mosaic TS. If the conditions are suitable for PGD, it may be beneficial to transfer embryos after PGD to patients with mosaic TS.

\section{Acknowledgments}

We thank participants, anonymous reviewers and the section editor for excellent criticism of the article.

\section{Conflict of interest}

The authors declare no conflict of interest.

\section{References}

1. Soenger P. Turner's syndrome. N Engl J Med 1996; 335: 1749-54.

2. Sybert VP. Phenotypic effects of mosaicism for a $47, \mathrm{XXX}$ cell line in Turner syndrome. J Med Genet 2002; 39: 217-20.

3. Abir R, Fisch B, Nahum R, Orvieto R, Nitke S, Ben Rafael Z. Turner's syndrome and fertility: current status and possible putative prospects. Hum Reprod Update 2001; 7: 603-10.

4. Bouchlariotou S, Tsikouras P, Dimitraki M, et al. Turner's syndrome and pregnancy: has the $45, \mathrm{X} / 47, \mathrm{XXX}$ mosaicism a different prognosis? Own clinical experience and literature review. J Matern Fetal Neonatal Med 2011; 24: 668-72.

5. Kaneko N, Kawagoe S, Hiroi M. Turner's syndrome - review of the literature with reference to a successful pregnancy outcome. Gynecol Obstet Invest 1990; 29: 81-7.

6. Tarani L, Lampariello S, Raguso G, et al. Pregnancy in patients with Turner syndrome: six new cases and review of literature. Gynecol Endocrinol 1998; 12: 83-7.

7. Borgstrom B, Hreinsson J, Rasmussen C, et al. Fertility preservation in girls with Turner syndrome: prognostic signs of the presence of ovarian follicles. J Clin Endocrinol Metab 2009; 94: 74-80.

8. Laranjeira C, Cardoso H, Borges T. Síndrome de Turner. Acta Pediatr Port 2010; 41: 38-43.

9. Gravholt $\mathrm{CH}$, Andersen NH, Conway GS. Clinical practice guidelines for the care of girls and women with Turner syndrome: pro- 
ceedings from the 2016 Cincinnati International Turner Syndrome Meeting. Eur J Endocrinol 2017; 177: G1-70.

10. Hovatta O. Ovarian function and in vitro fertilization (IVF) in Turner syndrome. Pediatr Endocrinol Rev 2012; 9: 713-7.

11. Bryman I, Sylven L, Berntorp K, et al. Pregnancy rate and outcome in Swedish women with Turner syndrome. Fertil Steril 2011; 95: 2507-10.

12. Hadnott TN, Gould HN, Gharib AM, Bondy CA. Outcomes of spontaneous and assisted pregnancies in Turner syndrome: the USNational Institutes of Health Experience. Fertil Steril 2011; 95: 2251-6.

13. Hagman A, Loft A, Wennerholm UB, et al. Obstetric and neonatal outcome after oocyte donation in 106 women with Turner syndrome: a Nordic cohort study. Hum Reprod 2013; 28: 1598-609.

14. Birkebaek NH, Cruger D, Hansen J, et al. Fertility and pregnancy outcome in Danish women with Turner syndrome. Clin Genet 2002; 61: 35-9. 